\title{
Riesgo de caída en el adulto mayor que acude a dos Centros de Día. Lima, Perú
}

Jack Roberto Silva-Fhon', Milagros Mariela Porras-Rodriguez ${ }^{2}$, Graciela Adilberta Guevara-Morote, Reyda Ismaela Canales-Rimachi ${ }^{4}$, Suzele Cristina Coelho Fabricio-Wehbe ${ }^{5}$, Rosalina Aparecida Partezani-Rodrigues ${ }^{6}$

\section{RESUMEN}

Objetivo: Evaluar el riesgo de caídas asociado a las variables sociodemográficas y el estado cognitivo en el adulto mayor que acude a dos Centros de Día en la ciudad de Lima - Perú

Material y Métodos: Estudio cuantitativo, descriptivo y de corte transversal; en una población de 150 adultos mayores de 60 años y más, de ambos sexos, que acuden a dos Centros de Día. Para la colecta de la información se utilizó los instrumentos de perfil social, Mini-Examen del Estado Mental y la Escala de Tinetti. Para el análisis de los datos se utilizó la estadística descriptiva. Análisis de asociación por el Odds Ratio con IC 95\% y significancia de $p<0.05$.

Resultados: Predominó el sexo femenino (75.3\%), el grupo etario con 80 años y más (33.3\%). A la evaluación de la Escala de Tinetti se obtuvo una puntuación promedio de $21.7(=6.1)$. 54.7\% presentó alto riesgo de caer; $36.7 \%$ con riesgo de caer con relación al estado cognitivo, se encontró que $20.7 \%$ presentaban estado cognitivo inadecuado. A la asociación, se verificó que el adulto mayor más viejo, presenta mayor riesgo de caer.

Conclusiones: Se resalta la necesidad que el equipo multi profesional incentive la actividad física para mantener una fuerza y postura adecuada, además de ejercicios de memoria preservando el estado cognitivo, promocionando un envejecimiento activo y saludable. (Horiz Med 2014; 14(3): 12-18)

Palabras clave: Desórdenes Cognitivos; Marcha; Balance postural; Anciano; Enfermería geriátrica. (Fuente: DeCS BIREME)

Risk of fall in the elderly to come to Day two Centers. Lima, Perú

\section{ABSTRACT}

Objective: Evaluate the risk of falls associated with sociodemographic variables and cognitive status in the elderly who came in to two day centers in Lima - Peru.

Material and Methods: A quantitative, descriptive, cross-sectional study; in a population of 150 adults age 60 and older of both sexes attending two day centers. For data collection we used the Instruments of social profile, Mini-Mental State Examination and the Tinetti Scale. For the analysis of the data we used descriptive statistics, for the association analysis we used the odds ratio with IC $95 \%$ and significance of $p<0.05$.

Results: Female prevalence was $75.3 \%$, the age group 80 years and over $33.3 \%$. In assessing the Tinetti Scale average was $21.7(=6.1)$ score, $54.7 \%$ also presented a high risk of falling; $36.7 \%$ risk of falling associated with congnitive function. In relation to cognitive status was found that $20.7 \%$ had inadequate cognitive status. The association was observed that the oldest the elderly patient, the greater risk of falling.

Conclusions: The necessity for the multidisciplinary team is highlighted, to encourages physical activity to maintain strength and proper posture, in addition to preserving the memory exercises cognitive state promoting an active and healthy aging. (Horiz Med 2014; 14(3): 12-18)

Key words: Cognition disorders; Gait; Postural balance; Aged; Geriatric nursing. (Source: MeSH NLM)

Magister en Ciencias; Doctorando de la Escuela de Enfermería de Ribeirão Preto - Universidad de São Paulo, Ribeirão Preto, Brasil; Asesor de Investigación de la Universidad Privada Norbert Wiener, Lima, Perú.

2 Licenciada en Enfermería; Escuela Académico Profesional de Enfermería - Universidad Privada Norbert Wiener, Lima, Perú.

3 Magister en Educación, Doctoranda de la Escuela de Post-Grado de la Universidad Norbert Wiener, Docente de la Escuela Académico Profesional de Enfermería - Universidad Norbert Wiener, Lima, Perú.

$4 \quad$ Magister en Gestión en Servicios de Enfermería, Doctoranda de la Escuela de Post-Grado de la Universidad Norbert Wiener, Docente de la Escuela Académico Profesional de Enfermería - Universidad Norbert Wiener, Lima, Perú.

5 Doctora en Enfermería Fundamental, Post-Doctoranda de la Escuela de Enfermería de Ribeirão Preto - Universidad de São Paulo, Ribeirão Preto, Brasil.

6 Doctora en Enfermería, Profesora Titular de la Escuela de Enfermería de Ribeirão Preto - Universidad de São Paulo, Ribeirão Preto, Brasil. 
Riesgo de caída en el adulto mayor que acude a dos Centros de Día.

Lima, Perú

\section{INTRODUCCIÓN}

El proceso de envejecimiento a nivel mundial, aumenta día a día. La Organización Mundial de la Salud (OMS) refiere que Latino-América será la región que más envejecerá en los próximos 50 años, debido a la disminución de la natalidad y a la mejora en la calidad de vida (1).

Con el proceso de envejecimiento, aparecen cambios biológicos que aumentan el riesgo de caer en el adulto mayor y es considerado un gran síndrome geriátrico (2).

La caída, es definida por la OMS, como cualquier acontecimiento en el cual el individuo se precipita al suelo en contra de su voluntad (3).

Entre las consecuencias que aparece posterior a la caída, está el miedo de sufrir una nueva caída, consecuentemente una disminución de las actividades habituales y pérdida de la independencia con aumento de la morbi mortalidad (4).

Un estudio brasilero, sobre el tema, con 240 adultos mayores que viven en comunidad, reportó que la prevalencia de caídas fue de $33.3 \%$, siendo que el $55.9 \%$ de los participantes que cayeron, reportaron que ésta se debió a una alteración en la marcha y en el equilibrio (5).

Del mismo modo, individuos que presentan alteración del equilibrio tienen $95.0 \%$ mayor prevalencia de caer comparado con aquellos que no presentan desequilibrio (6).

Los adultos mayores pueden presentar algún tipo de alteración en la marcha, que es definida, como el aumento de la susceptibilidad que tiene el adulto mayor de caer y que puede causar daños físicos. La alteración del equilibrio es denominado, como un fuerte indicador de riesgo de sufrir caídas que puede llevar a padecer limitaciones (7).

Otro cambio importante que ocurre en el adulto mayor, es la disminución del estado cognitivo, definido como cualquier déficit en las funciones mentales superiores tales como inteligencia, memoria y aprendizaje y puede estar influenciado por enfermedades crónicas degenerativas y aspectos psicosociales. Se estima que el $7 \%$ de los adultos mayores con 65 años y más, presentan algún grado de déficit llevándolo a la demencia y esta cifra aumenta a medida que avanza la edad (8).

Un estudio realizado en Egipto con 340 adultos mayores, encontró una prevalencia de caídas de $60.3 \%$, además, verificaron que aquel adulto mayor que presentaba déficit cognitivo tenía 1.97 veces mayor chance de caer comparado con aquél que tiene un estado cognitivo adecuado (4).

Existen otras afecciones que pueden llevar al adulto mayor a tener un alto riesgo de caída, tales como dolencias al oído: tipo otitis, otosclerosis; alteraciones visuales como cataratas, glaucoma y alteraciones de la musculatura cervical como el sufrir de artrosis (9).

El presente estudio, tuvo como objetivo evaluar el riesgo de caídas asociado a las variables sociodemográficas y el estado cognitivo en el adulto mayor que acude a dos Centros de Día en la ciudad de Lima - Perú.

\section{MATERIAL Y MÉTODOS}

El estudio fue de diseño cuantitativo, descriptivo de corte transversal (10) con adultos mayores de 60 años y más de ambos sexos que acudieron a dos Centros de Día en la ciudad de Lima - Perú.

Los Centros de Día, fueron concebidos como espacios de encuentro generacional de personas adultas mayores para mejorar el envejecimiento mediante el desarrollo de programas de soporte familiar, intergeneracional, sociocultural, productivo y programas de estilo de vida para un envejecimiento activo (11).

La población del estudio fue calculada por medio del paquete nQuery Advisor 7.0 considerando el análisis estadístico de correlación bivariada con un tamaño de efecto pequeño, nivel de significancia de 0.05 y potencia de $90 \%$ siendo la población calculada de 150 adultos mayores. 
Jack Roberto Silva-Fhon, Milagros Mariela Porras-Rodriguez, Graciela Adilberta Guevara-Morote, Reyda Ismaela Canales-Rimachi, Suzele Cristina Coelho Fabricio-Wehbe, Rosalina Aparecida Partezani-Rodrigues

La colecta de datos fue realizada entre los meses de Julio y Setiembre del 2012, por estudiantes de pre grado y profesores de enfermería capacitados. La entrevista tuvo una duración de 20 minutos aplicándose los siguientes instrumentos:

- Perfil demográfico: Para caracterizar al adulto mayor recogiendo información como: sexo, edad (años), tiempo que frecuentó el colegio (años) y si tiene o no pareja.

- Mini-Examen del Estado Mental (MEEM): Método práctico que permite establecer el grado del estado cognitivo del entrevistado, tiene un escore de 0 a 30 puntos. Validada al español tiene una sensibilidad de $80 \%$ y especificidad de $77.5 \%$ (12).

El punto de corte de 23 puntos para aquellos que tenían una escolaridad mayor de 5 años; de 21 puntos entre 1 a 4 años de estudios y de 17 puntos para aquel que no acudió al colegio; puntuaciones por debajo del punto de corte, indica que el adulto mayor presentaba un inadecuado estado cognitivo.

- Escala de Tinetti: Escala observacional que permite evaluar, a través de dos sub escalas, la marcha y el equilibrio, destinado para la evaluación de adultos mayores. Validada al español con una fiabilidad inter e intra observador de $0.4-0.6$ y $0.6-0.8$ y Alfa de Cronbach 0.91 (13).

La primera sub escala que explora el equilibrio dividido en estático y dinámico, y la segunda sub escala que evalúa la marcha, la suma total de ambas sub escalas es de 28 puntos; aquellos que obtienen un escore menor de 19 puntos, presentan un alto riesgo de caer; entre 20 a 23 puntos tienen riesgo de caídas y mayor a 24 puntos no presentan riesgo de caídas.

Para el análisis de los datos, se utilizó el programa Microsoft Excel $®$, se realizó doble digitación y validación de la información, para posteriormente importarlos al programa estadístico System Package for Social Sciences - SPSS v. 19.0.
Se realizaron análisis descriptivos, medida de tendencia central (media) y de dispersión (desviación estándar); para realizar el análisis de asociación (Odds Ratio) con intervalo de confianza del $95 \%$ y nivel de significancia de $p<0.05$.

El proyecto fue aprobado por el comité de investigación de la Escuela Académico Profesional de Enfermería de la Universidad Privada Norbert Wiener respetando los principios de la bioética de justicia, beneficencia, autonomía y la no maleficencia.

\section{RESULTADOS}

Se observó predominio del sexo femenino con $75.3 \%$; predominó el grupo etario de 80 años y más con $33.3 \%$; en relación a la escolaridad, se evidenció que $40.7 \%$ tuvo educación secundaria (11 años de estudio) y $58.7 \%$ no tenían pareja (Tabla 1 ).

Tabla 1. Perfil demográfico del adulto mayor que acude a dos Centros de Día. Lima, Perú.

\begin{tabular}{|c|c|c|c|c|c|}
\hline \multirow{2}{*}{\multicolumn{2}{|c|}{ Variable }} & \multirow{2}{*}{$\begin{array}{l}\text { Media } \\
(=\mathrm{ds})\end{array}$} & \multirow{2}{*}{$\begin{array}{c}\text { Rango } \\
{[\min -\text { máx }]}\end{array}$} & \multicolumn{2}{|c|}{ Total } \\
\hline & & & & $\mathbf{N}$ & $\%$ \\
\hline \multicolumn{6}{|l|}{ Sexo } \\
\hline & Masculino & & & 37 & 24.7 \\
\hline & Eemenino & & & 113 & 75.3 \\
\hline \multirow[t]{6}{*}{ Edad } & & $74.6(=7.2)$ & {$[60-91]$} & & \\
\hline & $60-64$ & & & 13 & 8.7 \\
\hline & $65-69$ & & & 26 & 17.3 \\
\hline & $70-74$ & & & 31 & 20.7 \\
\hline & & & & 30 & 20.0 \\
\hline & 80 y más & & & 50 & 33.3 \\
\hline \multicolumn{2}{|c|}{ Escolaridad } & $10.0(=4.5)$ & {$[0-30]$} & & \\
\hline & Analfabeto & & & 2 & 1.3 \\
\hline & Primaria & & & 41 & 27.3 \\
\hline & Secundaria & & & 61 & 40.7 \\
\hline & Superior & & & 46 & 30.7 \\
\hline \multicolumn{6}{|c|}{ Estado conyugal } \\
\hline & Con pareja & & & 62 & 41.3 \\
\hline & Sin pareja & & & 88 & 58.7 \\
\hline
\end{tabular}

A la evaluación de la marcha y del equilibrio por medio de la Escala de Tinetti, se encontró que de los 150 adultos mayores entrevistados la puntuación promedio alcanzada fue $21.7 \quad(=6.1)$ puntos e identificó que 37 (24.7\%) presentaron un riesgo alto 
de caer; 55 (36.7\%) fueron clasificados con riesgo de caer y $58(38.7 \%)$ no presentaban riesgo de sufrir caídas en todos los niveles con predominio del sexo femenino.

La evaluación del estado cognitivo por medio del MEEM, se obtuvo un promedio de $25.8(=4,3)$ puntos.
Además, se encontró que 31 (20.7\%) presentaban un estado cognitivo inadecuado y 119 (79.3\%) fueron clasificados como estado cognitivo adecuado.

Al análisis de regresión logística, se observó que el adulto mayor más joven (60 - 79 años) tiene 63\% de protección de no presentar riesgo de sufrir caída al compararlo con los adultos mayores más viejos (Tabla 2).

Tabla 2. Asociación entre el estado cognitivo y riesgo de caídas en el anciano que acude a dos Centros de Día. Lima, 2012.

\begin{tabular}{|c|c|c|c|c|c|c|c|}
\hline \multirow[t]{2}{*}{ Riesgo de caídas } & \multicolumn{2}{|c|}{ Sin riesgo } & \multicolumn{2}{|c|}{ Con riesgo } & \multirow[b]{2}{*}{$\mathbf{P}$} & \multirow[b]{2}{*}{ OR } & \multirow[b]{2}{*}{ IC (95\%) } \\
\hline & $\mathrm{n}$ & $\%$ & $\mathrm{n}$ & $\%$ & & & \\
\hline \multicolumn{8}{|l|}{ Sexo } \\
\hline Femenino & 46 & 40.7 & 67 & 59.3 & & 1 & \\
\hline Masculino & 12 & 32.4 & 25 & 67.6 & 0.80 & 0.90 & $0.39-2.07$ \\
\hline \multicolumn{8}{|l|}{ Edad } \\
\hline Adulto mayor más joven & 46 & 46.0 & 54 & 54.0 & & 1 & \\
\hline Adulto mayor más viejo & 12 & 24.0 & 38 & 76.0 & 0.01 & 0.37 & $0.17-0.83$ \\
\hline \multicolumn{8}{|l|}{ Estado civil } \\
\hline Con pareja & 23 & 37.1 & 39 & 62.9 & & 1 & \\
\hline Sin pareja & 35 & 39.8 & 53 & 60.2 & 0.61 & 1.19 & $0.59-2.40$ \\
\hline \multicolumn{8}{|l|}{ Estado cognitivo } \\
\hline Adecuado & 49 & 41.2 & 70 & 58.8 & & 1 & \\
\hline Inadecuado & 9 & 29.0 & 22 & 71.0 & 0.27 & 0.61 & $0.25-1.46$ \\
\hline
\end{tabular}

Prueba de regresión logística p $0.05^{*}$; OR: Odds Ratio

\section{DISCUSIÓN}

En el presente estudio, se evidenció que hubo una mayor prevalencia del sexo femenino; estudios internacionales $(14,15)$ y nacional $(16)$, encontraron datos similares: entre $56 \%$ a $68.3 \%$; esto puede deberse a que el sexo femenino tiene una mayor esperanza de vida que el masculino a nivel mundial, viviéndose actualmente el fenómeno de feminización en esta etapa de la vida.

Se evidenció que prevalecieron aquellos con una edad superior a los 80 años y más; estudios en esta población (14-17) encontraron también un mayor número de adultos mayores en este grupo etario; esto puede deberse a que la calidad de vida del anciano y la mejora del acceso a los servicios de salud se ha incrementado en las últimas décadas.
A nivel mundial, se observa un aumento en la esperanza de vida, en el Perú esta alcanza los 73 años; siendo para los hombres 70.5 y 75.9 años para las mujeres; el Instituto Nacional de Estadística e Informática (INEI) por medio de la Encuesta Nacional de Hogares (ENAHO) encontró en el segundo trimestre del 2012 que 9,2\% de la población total eran adultos mayores de los cuales $1.5 \%$ tenían 80 años y más, incrementándose $0.3 \%$ al realizar la comparación con el segundo trimestre del 2011 (18).

Un alto porcentaje acudió al colegio, solo 1.3\% no lo frecuentó, estudios revelan que esta prevalencia puede estar entre el 1,5 a 10,5\% (19,20); esta prevalencia puede deberse al tipo de población que se realizaron las diferentes investigaciones como áreas urbanas y rurales; los adultos mayores 
Jack Roberto Silva-Fhon, Milagros Mariela Porras-Rodriguez, Graciela Adilberta Guevara-Morote, Reyda Ismaela Canales-Rimachi, Suzele Cristina Coelho Fabricio-Wehbe, Rosalina Aparecida Partezani-Rodrigues

que acuden a los Centros de Día durante su vida productiva, requirieron de una mayor preparación educativa para acceder a un aseguramiento social y atendimiento de salud para él mismo y su familia.

Más de la mitad de los entrevistados no tenían pareja, diferente a un estudio realizado en el Brasil (14); esto podría deberse a que en el presente estudio prevaleció el sexo femenino y que además eran viudas.

A la evaluación de la marcha y del equilibrio, hubo una puntuación promedio de $21.7(=6.1)$ con un valor mínimo de 6 y máximo de 28 puntos; datos superiores fueron encontrados en un estudio con 20 adultos mayores, donde el promedio fue de 24 con un mínimo de 21 y máximo de 28 puntos; lo que se podría explicar porque algunos adultos mayores, utilizaban dispositivo de ayuda para deambular y su andar era lento (21).

24.7\% de adultos mayores presentaron un alto riesgo a caer, siendo mayor en el sexo femenino. Otro estudio en Perú (16), encontró resultados similares, siendo que el $21.0 \%$ de los participantes presentaron este riesgo; la literatura internacional refiere que aquellos adultos mayores con una puntuación menor de 19 puntos tienen un riesgo de caer 5 veces mayor $(22,23)$ lo que puede llevar al adulto mayor a limitaciones en la realización de las actividades de la vida diaria.

El riesgo de caídas a las que esta propenso esta población, puede deberse muchas veces al déficit visual, estudiosos del área, refieren que el déficit visual limita al anciano a realizar actividades y ver adecuadamente en lugares donde hay poca luz, distinguir la altura y objetos en el suelo (24).

Sufrir de enfermedades crónicas como alteraciones visuales, hipertensión, diabetes, enfermedades cardiovasculares, artritis y el consumo de múltiples medicamentos incrementan el riesgo de sufrir caídas $(25,26)$.

De otro lado, las personas al envejecer, se quejan en mayor frecuencia de olvidos cotidianos; este trastorno de la memoria relacionado con la edad es muy frecuente, sin embargo, los problemas de memoria con significación patológica son menos comunes.
A la evaluación con el MEEM, se evidenció que 21.3\% presentó déficit cognitivo, datos superiores se hallaron en un estudio con adultos mayores institucionalizados donde $39.3 \%$ (27), presentaron déficit cognitivo; esto se puede explicar ya que en los Centros de Día, el adulto mayor logra mantener un adecuado funcionamiento de la memoria ya sea por medio de actividades recreativas, ejercicios para la memoria como juego de casino, ajedrez, sudokus entre otras que los hace menos vulnerables a estos cambios fisiológicos (11).

La presencia de deterioro cognitivo puede ser un causante para sufrir de caídas siendo la segunda causa mundial de muerte por lesiones accidentales no intencionales; los adultos mayores son quienes corren mayor riesgo de muerte o lesión grave por caídas y este riesgo aumenta con la edad (3).

Se verificó que el adulto mayor más viejo (80 años o más) tuvo mayor riesgo de caer comparado con el adulto mayor más joven (60 - 79 años). Un estudio de seguimiento por tres años realizado en Australia con 69 adultos mayores encontró que el número de caídas aumento un $6 \%$ pudiéndose deber a una alteración en la marcha y en el avance de la edad observándose mayor caídas en los más viejos (28).

Con aumento de la edad, aumenta la probabilidad del adulto mayor de caer. Datos indican que el $30 \%$ de personas con edad entre los sesentas, el $40 \%$ en los setentas y $50 \%$ en los ochentas sufren más de una caída al año (29).

Se observó que entre la escala de Tinetti y el MEEM, el $71.0 \%$ presentó riesgo de caer y déficit cognitivo. Un estudio evaluando dos grupos de adultos mayores donde el primer grupo presentaba Alzheimer y el otro grupo no presentaba ninguna alteración mental, dio como resultado que en aquellos que tenían Alzheimer eran más propensos a sufrir alto riesgo de caída comparados a los que no presentaban déficit cognitivo (30).

Otros estudios revelan que las intervenciones sobre el estado cognitivo en el adulto mayor mejoran la función en la ejecución de actividades, desarrollando habilidades para prevenir las caídas; pero las personas con problemas de equilibrio pueden no ser susceptibles a estas intervenciones, 
pudiéndose utilizar otras terapias de prevención de caídas, como entrenamiento de la fuerza y del equilibrio $(31,32)$.

En conclusión, se observó que el adulto mayor más viejo, 80 años y más, tiene más riesgo de caer comparado con el adulto mayor más joven.

Para que los adultos mayores mantengan y mejoren su estado cognitivo sería necesario implementar programas educativos con un equipo multidisciplinario para los adultos mayores y familiares y/o cuidadores con el fin de estimular y mantener la memoria activa. Es preciso incentivar la actividad física por medio de ejercicios adecuados para proteger y conservar la marcha y el equilibrio previniendo complicaciones y disminuyendo la morbi-mortalidad, mejorando la calidad de vida e incentivando el envejecimiento saludable.

\section{Fuentes de financiamiento}

Universidad Privada Norbert Wiener; Universidad de São Paulo, apoyo logístico y Agencia financiera CAPES del Brasil.

\section{Conflictos de interés}

Los autores declaran no tener conflictos de interés.

\section{Correspondencia:}

Roberto Silva Fhon

Dirección: Rúa Machado de Assis 971 Bairro Vila

Tiberio CEP 14050-490, Ribeirão Preto, São Paulo -

Brasil

Teléfono: + 5516-981019136

Correo electrónico: beto_fhon@hotmail.com

\section{REFERENCIAS BIBLIOGRÁFICAS}

1. Organización Mundial de la Salud. [Internet]. Día Mundial de la Salud 2012 - La buena salud añade vida a los años [acceso en: 16 Dic 2013]. Disponible en: http://www.who.int/ageing/es/

2. Quintero CMV. Riesgo de caídas en las personas mayores institucionalizadas en hogares geriátricos del distrito de Barranquilla. Rev Salud Mov 2009;1(1):21-25.

3. Organización Mundial de la Salud. [Internet]. Caídas en el anciano [acceso en: 30 Dic 2013]. Disponible en: http://www.who.int/ mediacentre/factsheets/fs344/es/

4. Kamel MH, Abdulmajeed AA, Ismael SE-S, Risk factors of falls among elderly living in urban Suez - Egypt. Pan African Medical Journal 2013;14(26). Doi: 10.11604/pamj.2013.14.26.1609.

5. Fhon JRS, Rosset I, Freitas CP, Silva AO, Santos JLF, Rodrigues RAP. Prevalence of falls among frail elderly adults. Rev Saúde Pública 2013; 47(2):266-73.

6. Brito TA, Coqueiro RS, Fernandes MH, Jesus CS. Determinants of falls in Community-dwelling elderly: Hierarchical analysis. Public Health Nursing 2014; 31(4):290-297.

7. Russell MA, Hill KD, Blackberry I Day LL, Dharmage SC. Falls risk and functional decline in older fallers discharge directly from emergency departments. Journal of Gerontology, Medical Sciences 2006; 61A(10):1090-1095.

8. Potter PA, Perry AG. Valoración de las necesidades del anciano. In: Fundamentos de enfermería. Sexta edición., vol. 1, Madrid España: McGraw-Hill; 2008:426-442.

9. Soares AV. A contribuição visual para o controle postural. Rev Neurocienc 2010;18(3):370-379.

10. Hulley SB, Cummings SR, Browner WS, Grady DG, Newman TB. Delineando a pesquisa clínica. Tercera edición, Santa Catarina Brasil: Artmed; 2008:46-55.

11. Perú, Los centros del adulto mayor como modelo gerontológico social: la experiencia de EsSalud. Primera edición, Lima Perú: EsSalud; 2012;156.

12. Ostrosky F, López G, Ardila A. Sensitivity and specificity of the Mini Mental State Examination in a Spanish speaking population. Applied Neurophysiology 2000; 7(1):25-31

13. Rodríguez GC, Helena LL. Validez y confiabilidad de la escala de Tinetti para población colombiana. Rev Colomb Reumatol 2012; 19(4):218-233.

14. Fhon JRS, Fabricio-Wehbe SCC, Vendruscolo TRP, Stackfleth R, Marques S, Rodrigues RAP. Caídas en el adulto mayor y su relación con la capacidad funcional. Rev Latino-Am Enfermagem 2012; 20(5):927-34.

15. Lojudice DC, Laprega MR, Rodrigues RAP, Rodrigues Jr AL. Falls of institutionalized elderly: Occurrence and associated factors. Rev Bras Geriatr Gerontol 2010;13(3):403-412.

16. Ore TEA, Piñeyro QGJ. Riesgo de caídas en el adulto mayor según el Test de Tinetti: Hospital de Día de Geriatría del Hospital Nacional Guillermo Almenara Irigoyen Enero - Diciembre 2010 (Licenciado en Enfermería). Lima, Perú. Universidad Privada Norbert Wiener (Facultad Ciencias de la Salud), 2012. 86.
Recibido: 13 de Mayo de 2014 Aprobado: 12 de Agosto de 2014 
Jack Roberto Silva-Fhon, Milagros Mariela Porras-Rodriguez, Graciela Adilberta Guevara-Morote, Reyda Ismaela Canales-Rimachi, Suzele Cristina Coelho Fabricio-Wehbe, Rosalina Aparecida Partezani-Rodrigues

17. Giraldo $\mathrm{Cl}$, Franco AGM. Functional capacity and health: guidelines and nursing care of the elderly. Av. Enferm 2008;26(1):43-58.

18. Perú. Instituto Nacional de Estadística e Informática. Informe técnico No 04 de diciembre 2001. Situación de la niñez y del adulto mayor - Trimestre: Julio - Agosto - Setiembre 2011.

19. Aparicio LL, Delgado ZR. Estilo de vida y factores biosocioculturales del adulto mayor. Urbanización Casuarinas, Nuevo Chimbote, 2011. In Crescendo 2011; 2(1):131/146.

20. Zavala LGI. Nivel de satisfacción del paciente adulto mayor sobre el cuidado que brinda la enfermera en el Servicio de Medicina del Hospital Nacional Dos de Mayo en el 2009 (Licenciada en Enfermería). Lima, Perú: Universidad Nacional Mayor de San Marcos, 2010. 96.

21. Piosevan AC, Pivetta HMF, Peixoto JMB. Predisposing factors for falls in the elderly residents in western region of Santa Maria, RS. Rev Bras Geriatr Gerontol 2011;14(1):75-83.

22. Brasil, Ministério de Saúde Brasil. Envelhecimento e saúde da pessoa idosa. 2007.

23. Maita RAF. Riesgos de accidentes en el adulto mayor que realiza actividades de rutina, usuarios de los servicios del Centro de Salud Conde de la Vega Baja, Lima 2008. (Licenciada en Enfermería). Lima, Perú. Universidad Nacional Mayor de San Marcos, 2009. 95.

24. Soares AV. A contribuição visual para o controle postural. Rev Neurocienc 2010;18(3):370-379.

25. Chang ChM, Chen MJ, Tsai ChY, Ho LH, Hsieh HL, Chau YL et al. International Journal of Geriatric Psychiatry 2011;26(6):602-607.
26. Chen TY, Peronto CL, Edwards JD. Cognitive function as a prospective predictor of falls. The Journal of Gerontology, Series B: Psychological Sciences and Social Sciences 2012; 67(6):720-728.

27. Mello BLD, Haddad MCL, Dellaroza MSG. Cognitive evaluation of institutionalized elderly. Acta Scientiarum 2012;34(1):95-102.

28. Bird ML, Pittaway JK, Cuisick I, Rattray M, Ahuja KDK. Age-related changes in physical fall risk factors: results from 3 year follow-up of communitty dwelling older adults in Tasmania, Australia. Int. J. Environ. Res. Public Health 2013;10:5989-5997.

29. Campbell, A.; Borrie, M.J.; Spears, G.F.; Jackson, S.L.; Brown, J.S.; Fitzgerald, J.L. Circumstances and consequences of falls experienced by a community population 70 years and over during a prospective study. Age Ageing 1990; 19:136-141.

30. Kato-Narita EM, Nitrini R, Radanovic M. Assessment of balance in mild and moderate stages of Alzheimer disease: Implications on falls and functional capacity. Arq Neuropsiquiatr 2011; 69(2A):202

31. Buracchio TJ, Mattek NC, Dodge HH, Hayes TL, Pavel M, Howieson $\mathrm{DB}$ et al. Executive function predicts risk of falls in older adults without balance impairment. BMC Geriatrics 2011; 11:74. Doi: $10.1186 / 1471-2318-11-74$

32. Winter $\mathrm{H}$, Watt $\mathrm{K}$, Peel NM. Falls prevention interventions for community-dwelling older persons with cognitive impairment: sistematic review. International Psychogeriatrics 2013; 25(2):215227

\section{HORIZONTE MÉDICO} Indizada a: Scielo, Latindex, DOAJ, entre otras

\section{Difundiendo la investigación, más allá; de nuestras fronteras}

Se distribuye gratuitamente y por canje.

Está disponible a texto íntegro en el siguiente e-mail: horizonte_medico@usmp.pe y

en la web: www.medicina.usmp.edu.pe/horizonte/principal.php

Síguenos en:

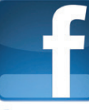

facebook 